\title{
Evaluation of the Behaviour of Wrinkles Fibroblasts and Normal Aged Fibroblasts in the Presence of Poly-L-Lactic Acid
}

\author{
Carol Courderot-Masuyer ${ }^{1}$, Sophie Robin ${ }^{1}$, Hélène Tauzin ${ }^{1,2}$, Philippe Humbert ${ }^{2,3,4,5^{*}}$ \\ ${ }^{1}$ Bioexigence SARL, Espace Lafayette, Besançon, France; ${ }^{2}$ Department of Dermatology, Laboratory of Cutaneous Biology, Besan- \\ çon, France; ${ }^{3}$ Clinical Investigation Center, Besançon, France; ${ }^{4}$ University of Franche-Comte, Besançon, France; ${ }^{5}$ INSERM \\ UMR1098, SFR FED 4234 IBCT, Besançon, France. \\ Email: "
}

Received November $8^{\text {th }}, 2011$; revised November $27^{\text {th }}, 2011$; accepted December $8^{\text {th }}, 2011$

\begin{abstract}
Background: Wrinkles are characterized by changes in the organization and structure of the dermis. Human wrinkle fibroblasts (WF) have a different functional behaviour in comparison with normal-aged fibroblasts (NF). Decreases in migration capacities and collagen I synthesis are observed. Mitochondrial function is impaired with an increase in lactate production during aging. Sculptra ${ }^{\circledR}$ (poly-L-lactic acid: PLLA), a biodegradable synthetic polymer, is used for subcutaneous volume restoration. Thus we decided to investigate different fibroblast functions when placed in contact with PLLA. Objectives: The potential of PLLA to compensate for the reduction of metabolic activity, to restore the migration capacity of WF and to inhibit the lactate production, was investigated and compared to NF. Methods: Two different skin samples were used from each of the three women's facelift (one inside a face wrinkle and one from normal aged skin). Collagen I, lactate productions and proliferation capacities were investigated on monolayer cultures. Migration properties were evaluated using three-dimensional collagen lattices. Results: PLLA increased collagen I synthesis, restored migration capacities and tended to decrease lactate production in WF, whereas PPLA stimulated proliferation in NF and tended to improve the migration of NF. Conclusion: These results suggested that PLLA from Sculptra ${ }^{\circledR}$ acted as a stimulus for collagen production in WF and that it is suitable for correcting skin depressions, such as wrinkles.
\end{abstract}

Keywords: Wrinkles; Poly-L-Lactic Acid; Collagen; Fibroblast

\section{Introduction}

Injectable Poly-L-lactic acid (PLLA) was introduced into Europe in 1999 as New-fill ${ }^{\circledR}$, receiving European Union approval to increase the volume of depressed areas, such as skin creases, wrinkles, folds and scars. In February of 2004 , this indication was extended to include largevolume corrections of lipoatrophy, and in the same year it received U.S. Food and Drug Administration approval for the restoration and/or correction of the signs of facial lipoatrophy in people with human immunodeficiency virus [1]. Its cosmetic use was approved by the FDA in 2009 [2] and relies upon volumetric expansions as well as the formation of new collagen and elastic fibers. Thus, the gradual increase in dermal thickness in patients receiving PLLA injections is thought to be due to the induction of a local tissue reaction such as acute inflammatory reaction. This effect is characterized by a foreign body reaction that includes an initial increase in fibro

*Corresponding author. blast activity and a subsequent progressive increase in collagen fiber deposition over the course of weeks to months $[3,4]$. Previous studies have demonstrated a particular behavior of WF different from that of NF. These fibroblasts populations were obtained after punch biopsies performed inside a wrinkle present on discarded tissues from a facelift and also on surrounding normal aged skin. After a comparison of these two populations of fibroblasts, a significant decrease in collagen I production and a significant impairment of migratory capacities were observed in WF compared to NF from the same patient [5]. Reduced synthesis of collagen I is partly responsible for the aging of the skin because it confers resistance to stresses and traction. The reduction in collagen I synthesis was also demonstrated by a lower in vitro production of type I procollagen by dermal fibroblasts isolated from skin of old versus young individuals. There is a failure to replace damaged collagen I with newly synthesized material [6]. To our knowledge, no study was performed in order to evaluate the effect of PLLA on 
the behavior of specific WF. During aging, an alteration of mitochondrial respiration is often associated with increased lactates production [7]. Indeed it was observed that senescent skin fibroblasts displayed higher rates of utilization of glucose and amino acids and produced more pyruvate and lactate, compared with young skin fibroblasts [8]. We therefore decided to investigate the effects of PLLA on the production of lactates in WF compared to NF. In the present study, the effects of PLLA $\left(0.1 \%\right.$ Sculptra $\left.{ }^{\circledR}\right)$ on proliferation, migratory capacities, collagen I and lactates production of WF compared to those of NF from the same patients were investigated.

\section{Materials and Methods}

\subsection{Products}

Vials of dry powder of Sculptra ${ }^{\circledR}$ (poly-L-Lacid acid, Sanofi aventis, France) were used in the present study. 5 $\mathrm{ml}$ of sterile water were added to the dry powder of Sculptra ${ }^{\circledR}$ and $0.1 \%$ of this solution was added to culture medium of fibroblasts.

\subsection{Fibroblast Cultures}

Punch biopsies were performed inside a wrinkle and also on surrounding normal aged skin of patients after surgical procedure. Wrinkles fibroblasts (WF) and normal aged fibroblasts (NF) were obtained from discarded tissues from three healthy female patients who had given their written consents (aged 55, 49 and 50 years old). Three fibroblast lines of WF and NF were obtained: L1 (55 years), L2 (49 years) and L3 (50 years). Cells were maintained in Dulbecco's Modified Eagle's Medium (DMEM) supplemented with $10 \%$ foetal calf serum (FCS) and $40 \mathrm{mg} / \mathrm{ml}$ of gentamicin and $2 \mathrm{mg} / \mathrm{ml}$ of fungizone (DMEMc). They were grown for several weeks in plastic flasks in a moist atmosphere of 5\% carbon dioxin and $95 \%$ air at $37^{\circ} \mathrm{C}$. Confluent monolayers were propagated by trypsination (trypsin-EDTA $1 \mathrm{X}$ solution, Gibco) and replated at 1:2 dilutions. Subcultures of fibroblasts over 10 were not used.

Different groups were used for each experiment:

- NF: normal aged fibroblasts $(n=6)$

- NF + PLLA $\left(0.1 \%\right.$ Sculptra $\left.^{\circledR}\right)(n=6)$

- WF: wrinkle fibroblasts $(n=6)$

- $\mathrm{WF}+\operatorname{PLLA}\left(0.1 \%\right.$ Sculptra $\left.^{\circledR}\right)(\mathrm{n}=6)$

\subsection{Fibroblasts Proliferation}

Fibroblast proliferation in monolayers was assessed using the 3-(4,5-dimethylthiazol-2-yl)-2,5-diphenyltetrazolium bromide (MTT) dye-reduction assay after the action of PLLA for 24 hours, 48 hours and 72 hours. All cul- tures were set up in 10 wells. Fibroblasts were placed in 96-well microliter plates at a cell density of $5 \times 10^{3}$ cells per well. After 24 hours of incubation, PLLA was added to the culture medium of WF or NF. The fibroblasts were seeded with PLLA, one for 24 hours, one for 48 hours and the final one for 72 hours. Then MTT was added. The extraction buffer was added after 4 hours. Absorbance of each well was assessed using a Multiscan Ex (Thermo, France) equipped with a $550 \mathrm{~nm}$ filter.

\subsection{Migratory Capacity}

$\mathrm{NF}$ and WF were embedded in three-dimensional hydrated collagen using a modified version of the technique developed by Bell et al. [9]. Collagen lattices were made by mixing: six volumes of $1.76 \mathrm{X}$ concentrated medium, three volumes of rat tail type I collagen solution (2 $\mathrm{mg} / \mathrm{ml})$ and one volume of fibroblast suspension $\left(8 \cdot 10^{5}\right.$ cells $/ \mathrm{ml})$. This mixture was poured into plastic Petri dishes $(60 \mathrm{~mm}$ diameter) $(\mathrm{n}=10)$. The gel polymerized in less than 10 minutes at $37^{\circ} \mathrm{C}$. After one hour, culture medium was added and changed every 48 hours. To measure the retracted lattice diameter, the culture dishes were placed on a transparent metric scale. The measurement of the lattice diameter was taken each day for 10 days.

\subsection{Collagen I Synthesis}

Fibroblasts were seeded in 12-well plates at $0.04 \times 10^{6}$ cells per well. The plates were incubated at $37^{\circ} \mathrm{C}$ with and without the presence of PLLA in DMEMc with $1 \%$ foetal calf serum for 7, 21, 30 and 42 days respectively (n $=6$ ). The culture medium was changed twice a week. After this period, the supernatant was placed in an antiprotease solution $(10 \% \mathrm{v} / \mathrm{v}$, Calbiochem, France). The samples were stored at $-80^{\circ} \mathrm{C}$ until the analysis by Elisa method was performed. Collagen I was evaluated using murine anticollagen I monoclonal antibody (5). The antimurine second antibody was peroxidase conjugated (Sigma-Aldrich, France). Color was developed using a TMB substrate. The absorbance was read by a spectrophotometer at $620 \mathrm{~nm}$ (Multiscan, Thermo, France). The number of cells was counted and the amount of collagen I was expressed as $\mu \mathrm{g}$ of collagen I/million of cells. Different groups were used at $\mathrm{T}=0,7,21,30$ and 42 days of fibroblast cultures.

\subsection{Lactate Production}

Fibroblasts were seeded in 12 -wells plates at $0.04 \times 10^{6}$ cells per well. The plates were incubated at $37^{\circ} \mathrm{C}$ both with and without the presence of PLLA for 7, 21, 30 and 42 days of fibroblast cultures $(n=6)$. The culture me- 
dium was changed twice a week. After the incubation period, the supernatant was placed in an antiprotease solution $(10 \%, v / v$, Calbiochem, France). The samples were stored at $-20^{\circ} \mathrm{C}$ until the analysis. The dosage of lactates was performed by the biochemistry department of St Jacques hospital (Besançon, France) using Dimen$\operatorname{sion}^{\circledR}$ system. The oxidation of lactate in pyruvate with reduction of NAD is obtained with rabbit lacticodeshydrogenase. One molecule of NAD is transformed into one molecule of NADH for each molecule of lactate. Absorbance of NADH is proportional to the concentration of lactate and measured by spectrophotometry (340 $\mathrm{nm})$. The number of cells is counted and the amount of lactates is expressed as $\mu$ moles of lactates/million of cells.

\subsection{Statistical Analysis}

The results shown were expressed as the mean of the obtained results with the 3 cell lines (L1, L2 and L3).

The groups with PLLA and those without PLLA were compared by using one way (for cell proliferation) or two ways (for the other experiments) variance analysis followed if necessary by Fisher test. Values were considered significantly different when $\mathrm{p}<0.05$.

\section{Results}

\subsection{Cell Proliferation}

The viability of NF and WF of the 3 women was higher than $80 \%$ in the presence of PLLA $\left(0.1 \%\right.$ of Sculptra $\left.{ }^{\circledR}\right)$ providing the chosen dose for the following experiments. PLLA induced a cell proliferation in NF after 72 hours of culture (Figure 1).

\subsection{Migration Capacity}

The migration of fibroblasts was evaluated through their capacity to retract free floating collagen lattices. The retraction of collagen lattices obtained with normal aged fibroblasts depended on the time (a decrease of $68 \%$ from the initial diameter was observed at the end of 10 days). A significant difference was observed between the retraction of collagen lattices obtained with NF and WF. The cell migration was slower with WF than NF. The presence of PLLA tended to improve the retraction of collagen lattices from NF (the difference was significant for cell lines L1 and L2) suggesting a beneficial effect of PLLA on migratory capacities of NF (Figure 2). PLLA restored the migration capacities of WF to the same level as basal values of NF.

\subsection{Collagen I Production}

Figure 3 shows the basal amount of collagen I in NF and WF groups (D0). The production of collagen I was significantly lower in WF than in NF. PLLA increased the mean production of collagen I of WF after 42 days of culture but did not modify the collagen I production of NF (Figure 4).

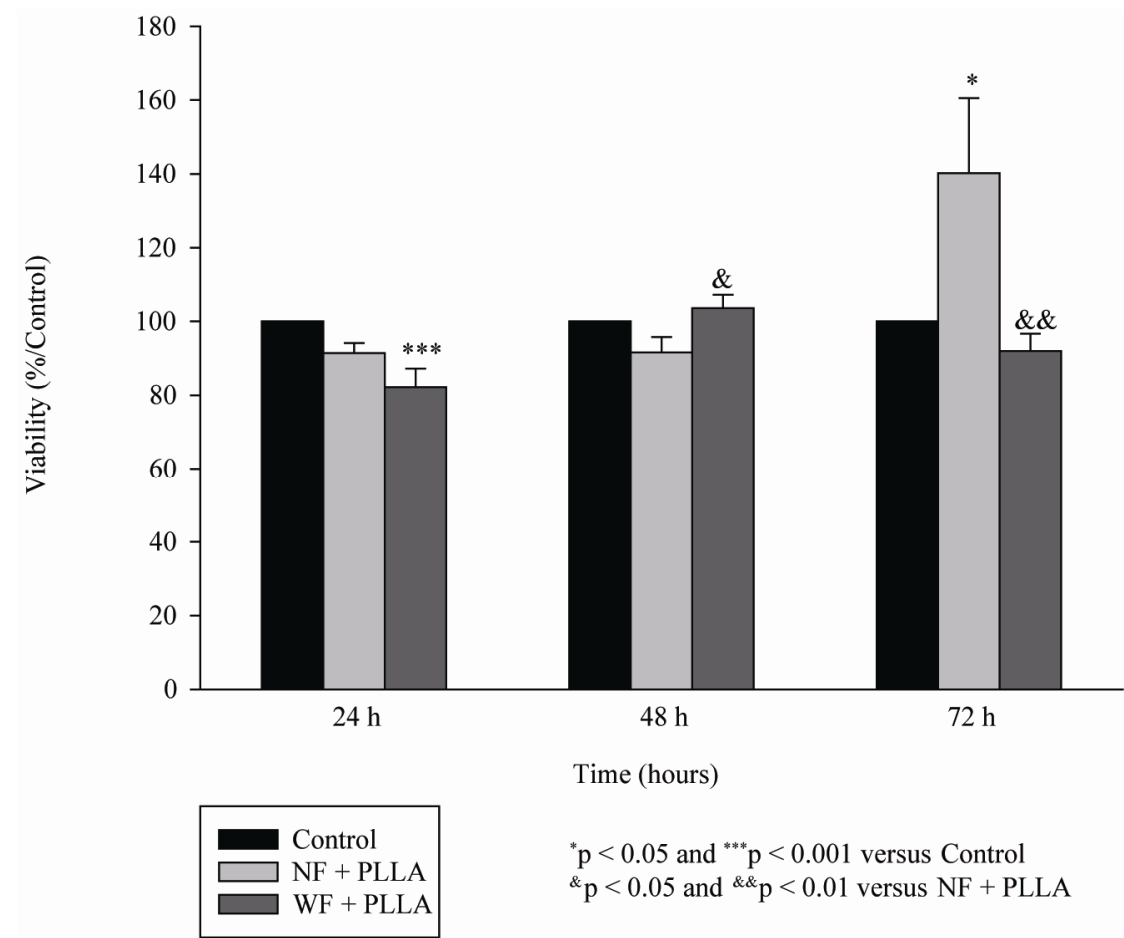

Figure 1. Proliferation of WF $(n=3)$ or NF $(n=3)$ after 24, 48 and 72 hours of culture in the presence of PLLA. 


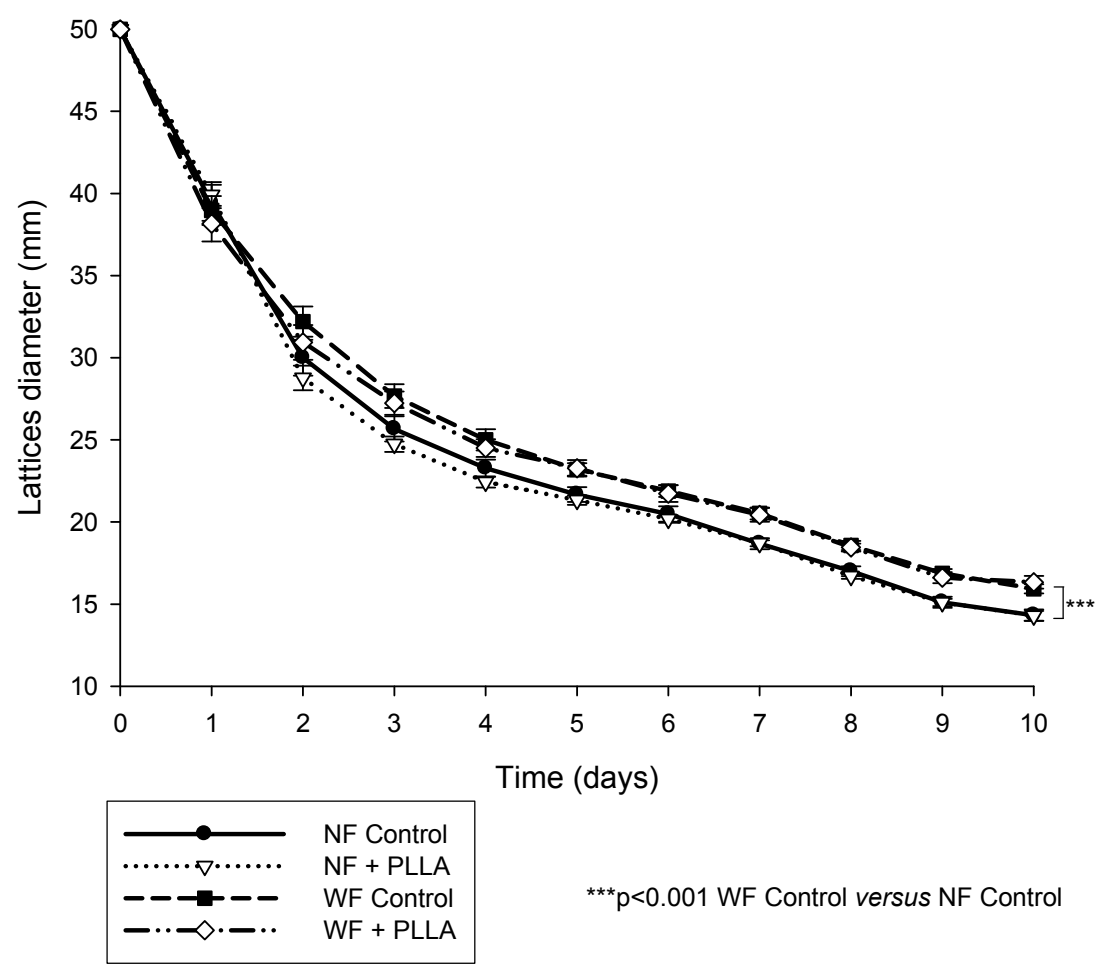

Figure 2. Mean of lattices diameter of WF $(n=3)$ or NF $(n=3)$ in the presence of PLLA.

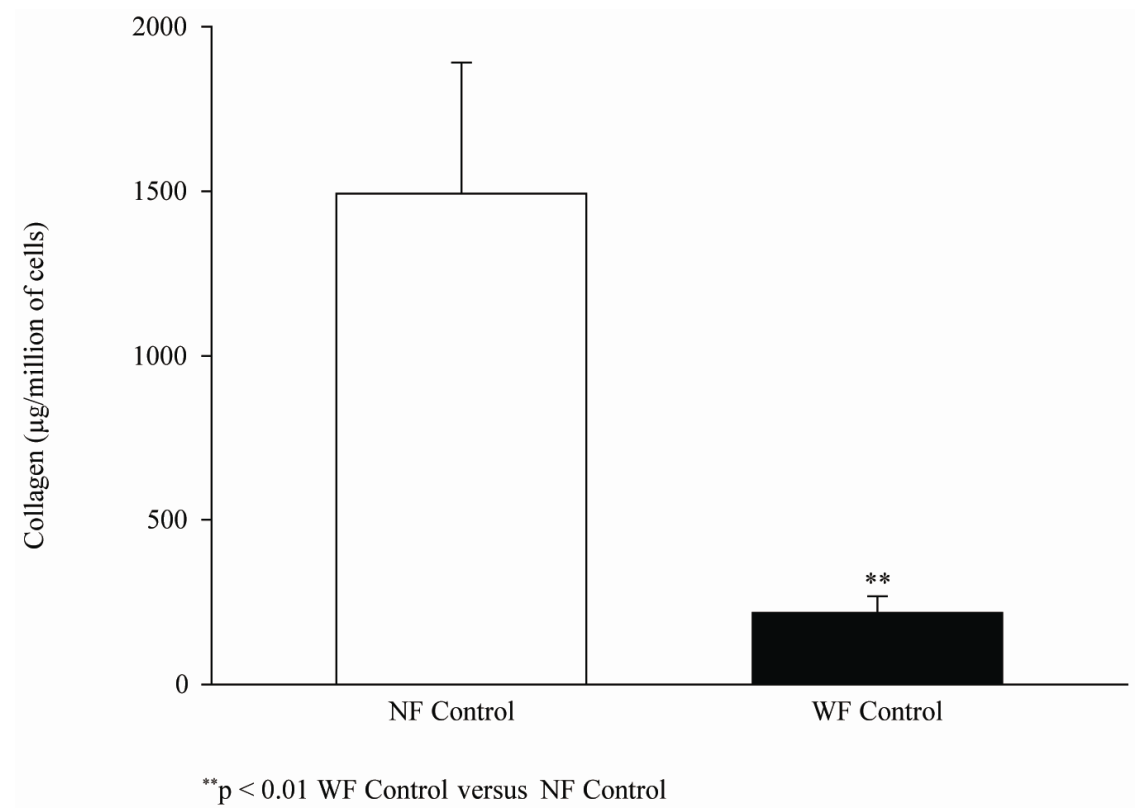

Figure 3. Mean basal Collagen I synthesis on D0 of WF or NF.

\subsection{Lactate Production}

The amount of lactate was significantly higher in WF than in NF after 30 and 42 days of culture. There was not a statistically significant decrease in lactates in WF in the presence of PLLA but there was a trend for a reduction in lactate production with 2 individual cell lines (L1 and L3 showing a significant decrease). The presence of PLLA in the culture medium of NF did not affect its lactate production level (Figure 5).

\section{Discussion}

Wrinkles are one of the main expressions of skin aging 


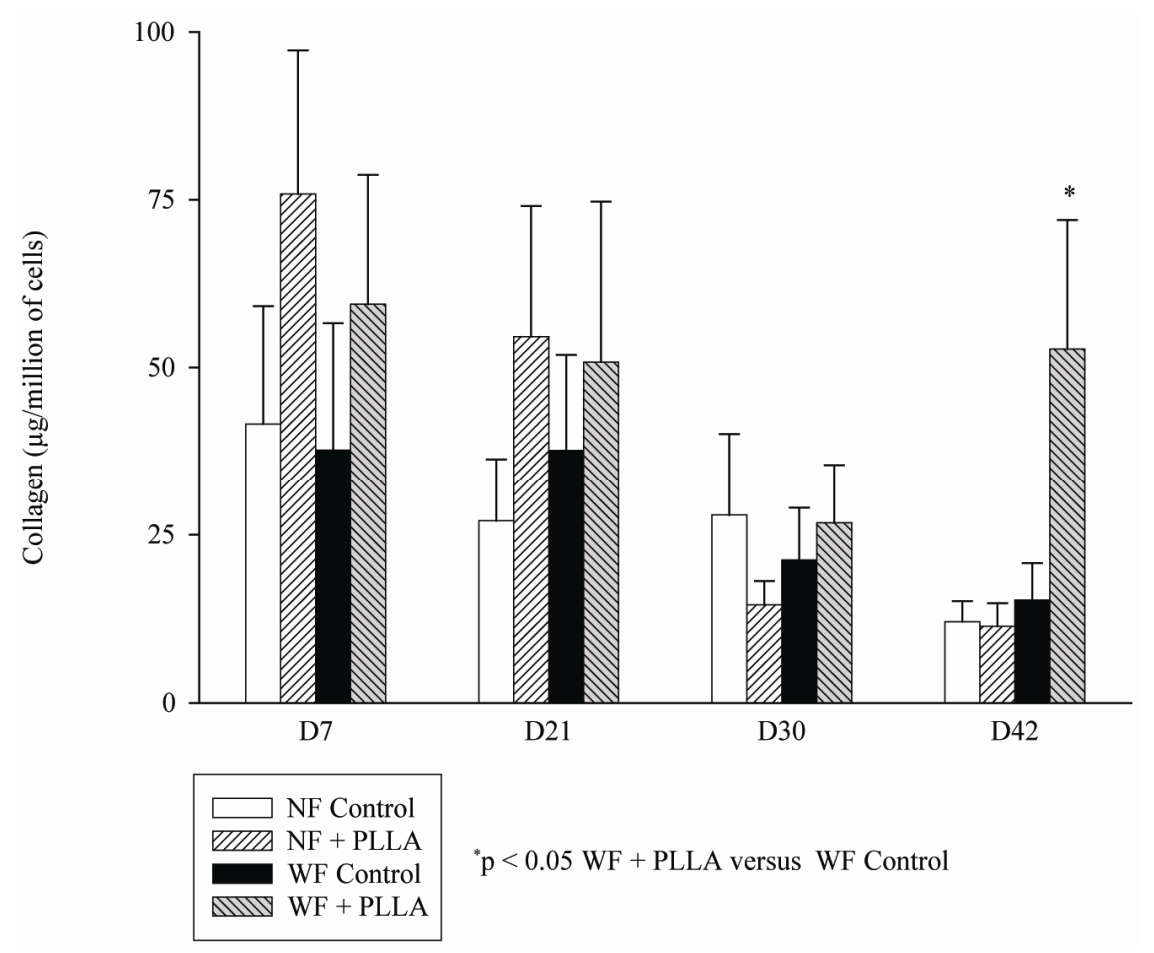

Figure 4. Collagen content of WF $(n=3)$ or NF $(n=3)$ from D7 to D42 with and without the presence of PLLA.

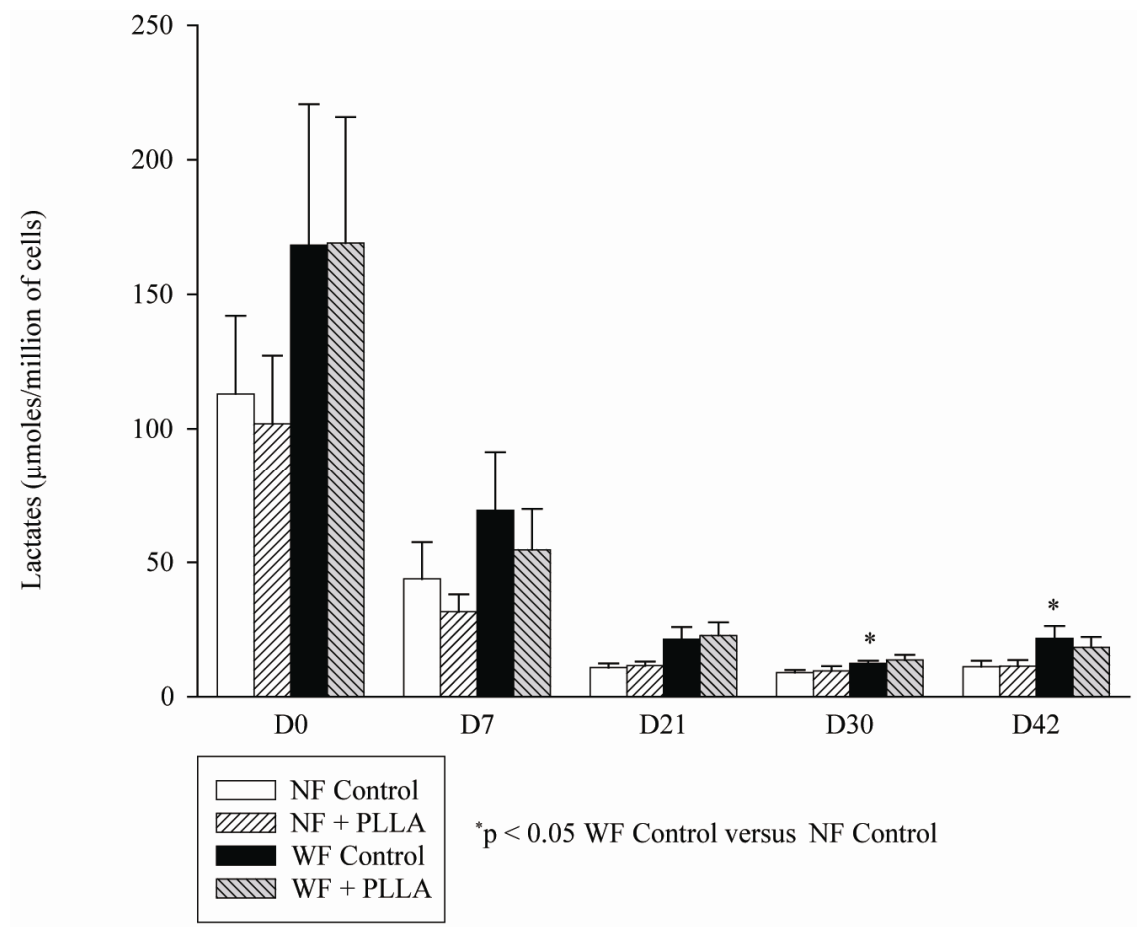

Figure 5. Mean lactate production of WF $(n=3)$ or NF $(n=3)$ in the presence of PLLA from D0 to D42.

and are characterized by numerous alterations in dermis organization and structure [6]. During chronological aging, the dermis becomes thinner as the dermo-epidermal junction flattens and the stratum corneum loses its integ- rity. Dermal content in collagen is diminished [10], collagen meshwork tends to disappear and therefore interactions between fibroblasts and collagen are reduced leading to randomly oriented collapsed fibroblasts [6]. These 
alterations are due to a weaker procollagen synthesis such as procollagen I by the fibroblasts [11] and a slight increase in collagen degradation by active MMPs [12]. In addition to these changes, the more substantial volume loss and predominant source of facial wrinkles and folds are not only derived from lipoatrophy and an overall fat redistribution associated with aging, but also related to repeated muscle contraction.

PLLA has been used for several years in Europe. It received approval from the Food and Drug Administration for the treatment of HIV-associated lipoatrophy in $2004[13,14]$ and for cosmetic use in 2009 [2]. Sculptra ${ }^{\circledR}$ is a volumizer lasting 18 to 24 months [15]. Sculptra ${ }^{\circledR}$, longetivity is based on the low degradation kinetics of the PLLA microparticles, a result of irregularly shaped microparticles $[3,16]$. The gradual increase in dermal thickness in patients receiving PLLA injections is thought to be due to the induction of a local tissue reaction. This effect is characterized by a foreign body reaction that includes an initial increase in fibroblasts and a subsequent progressive increase in collagen fiber deposition over the course of weeks to months [17-19]. Indeed when PLLA is injected, it causes an immediate tissue expansion (due to the fluid carrier) and physical improvement to the appearance of the skin which is purely mechanical. Once the carrier solution is reabsorbed, a slow process of biodegradation of the microspheres occurs. The process of hydration, loss of cohesion and molecular weight, solubilization and phagocytosis of PLLA by the host's macrophage [20] slowly degrades the PLLA into lactic acid microspheres and eliminates $\mathrm{CO}_{2}$ (via lactate/pyruvate route) by way of respiratory excretion, thus leaving behind the crystals to stimulate collagen $[4,17,19]$.

Previous studies suggested differences in biomechanical behavior and synthesis capacities between NF and WF. Cell movement in vitro has been reproduced in fibroblast-populated collagen lattices. Indeed NF suspended in a collagen gel can retract and reorganize collagen fibers. Fibroblasts reduce their proliferation potential and three mechanisms seem to be responsible for lattice contraction: cell contraction, cell tractional forced related to locomotion and cell elongation [21]. In our free floating collagen lattices formed with WF, reduced contractile capacities were observed compared to NF and these results are in accordance with previous studies [5]. The rate of collagen I synthesized by WF was lower than that of NF, these results are also in accordance with other studies [5]. During the cutaneous aging process, the mitochondrial respiratory chain function is altered, and an increase in lactate production is observed [22]. Lactates increase proton concentration, so the $\mathrm{pH}$ decreases. A decrease in $\mathrm{pH}$ can provoke a decrease in myosin ATPase activity through a competition mechanism between
$\mathrm{Ca}^{2+}$ and $\mathrm{H}^{+}$. The enzyme myosin light chain kinase catalyzes the phosphorylation of the 20,000 dalton light chain of myosin which causes cycloskeleton actin-myosin contraction and therefore fibroblast-populated collagen lattices contraction [23]. This enzyme requires $\mathrm{Ca}^{2+}$-calmodulin for its activity [24]. These changes may be responsible for the reduction in WF contraction in free floating collagen lattices because an increase in lactate production was observed in WF compared to NF. However the decreasing trend in lactate production observed during PLLA treatment, did not improve the cell migration in WF groups. Only NF exhibited an increase in cell migration in the presence of PLLA (observed in 2 of 3 cell lines). Calcium is a well-known factor in collagen synthesis [25]. The cytoskeleton is involved in the regulation of collagen synthesis and secretion. Microtubules are involved in transcellular movements of procollagen. Indeed agents which disrupt microtubular function inhibit the conversion of procollagen to collagen and secretion of procollagen [26]. Actin and myosin interact in the presence of ATP to generate movement. A decrease in the activity of myosin ATPase $\left(\mathrm{Ca}^{2+}\right.$-calmodulin dependent) could be linked to the decrease in $\mathrm{pH}$ [27]. So the low pH (due to high levels of lactates in WF) might also be responsible for the decrease in collagen I synthesis in WF compared to NF because the movement and the secretion of collagen require energy used by the interaction between actin and myosin [28]. Our results suggest that PLLA, through the decrease in lactate production, could induce collagen I synthesis in WF after 42 days of treatment. The fact that PLLA did not modify the migratory capacities of WF could be explained by the results of Bell et al. obtained with normal fibroblasts [9]. Bell demonstrated that, in normal fibroblasts, an increase in collagen quantity could decrease the retraction of collagen lattices. In our study, high level of collagen I content in WF was noted after 42 days of PLLA treatment suggesting an effect of PLLA on synthesis capacities of WF. After 10 days of treatment with PLLA, the migration capacities of WF were restored to the same level as the basal values of NF. It was noted that the content in collagen I of NF was not modified and the treatment with PLLA for 10 days tended to increase the retraction of collagen lattices. Indeed statistically significant alterations in 2 of 3 cell lines were observed after PLLA treatment in our study. It is noteworthy that differences in PLLA response in patients were observed in vivo [29]. This individual variability could also explain the increasing trend in migratory capacities of NF and the decreasing trend in lactate production in WF observed in the presence of PLLA. The present study has shown that PLLA is able to increase the production of collagen I, to restore migration capacities and tends to decrease lactate 
production in WF. To our knowledge, it is the first study investigating the direct effects of PLLA on human WF. Its possible direct metabolic effect through lactate production on WF enhances the filler action of poly-L-lactic acid.

\section{REFERENCES}

[1] C. M. Burgess and R. M. Quiroga, "Assessment of the Safety and Efficacy of Poly-L-Lactic Acid for the Treatment of HIV-Associated Facial Lipoatrophy," Journal of the American Academy of Dermatolology, Vol. 52, No. 2, 2005, pp. 233-239. doi:10.1016/j.jaad.2004.08.056

[2] R. Fitzgerald and D. Vleggaar, "Using Poly-L-Lactic Acid (PLLA) to Mimic Volume in Multiple Tissue Layers," Journal of Drugs in Dermatology, Vol. 8, No. 10, 2009, pp. s5-s14.

[3] D. Vleggaar and U. Bauer, "Facial Enhancement and the European Experience with Sculptra (Poly-L-Lactic Acid)," Journal of Drugs in Dermatolology, Vol. 3, No. 5, 2004, pp. 542-547.

[4] J. K. Wildemore and D. H. Jones, "Persistent Granulomatous Inflammatory Response Injected by Injectable Poly-L-Lactic Acid for HIV Lipoatrophy," Dermatologic Surgery, Vol. 32, No. 11, 2006, pp. 1407-1409. doi:10.1111/j.1524-4725.2006.32313.x

[5] M. Jouandeaud, C. Viennet, S. Bordes, B. Closs and P. Humbert, "Comparison of the Biomechanical and Biosynthetic Behavior of Normal Human Fibroblasts and Fibroblasts from Forehead Wrinkle," IFSCC Magazine, Vol. 7, No. 2, 2004, pp. 2-6.

[6] J. Varani, M. K. Dame, L. Rittie L, S. E. Fligiel, S. Kang, G. J. Fisher and J. J. Voorhees, "Decreased Collagen Production in Chronologically Aged Skin: Roles of AgeDependent Alteration in Fibroblast Function and Defective Mechanical Stimulation," American Journal of Pathology, Vol. 168, No. 6, 2006, pp. 1861-1868. doi:10.2353/ajpath.2006.051302

[7] Y. H. Wei, S. B. Wu, Y. S. Ma and H. C. Lee, "Respiratory Function Decline and DNA Mutation in Mitochondria, Oxidative Stress and Altered Gene Expression during Aging," Chang Gung Medical Journal, Vol. 32, No. 2, 2009, pp. 113-132.

[8] W. Zwerschke, S. Mazurek, P. Stöckl P, E. Hütter, E. Eigenbrodt and P. Jansen-Dürr, "Metabolic Analysis of Senescent Human Fibroblasts Reveals a role for AMP in Cellular Senescence," The Biochemical Journal, Vol. 376, No. 2, 2003, pp. 403-411. doi:10.1042/BJ20030816

[9] E. Bell, B. Ivarsson and C. Merill, "Production of a Tissue-Like Structure by Contraction of Collagen Lattices by Fibroblasts of Different Proliferative Potential in Vitro," Proceedings of the National Academy of Sciences of the USA, Vol. 76, No. 3, 1979, pp. 1274-1278. doi:10.1073/pnas.76.3.1274

[10] R. M. Lavker, P. S. Zheng and G. Dong, "Morphology of Aged Skin," Clinics in Geriatric Medicine, Vol. 5, No. 1, 1989, pp. 53-67.
[11] J. Uitto, "Connective Tissue Biochemistry of the Aging Dermis. Age-Associated Alteractions in Collagen and Elastin," Clinics in Geriatric Medicine, Vol. 5, No. 1, 1989, pp. 127-147.

[12] J. Varani, G. J. Fisher, S. Kang and J. J. Voorhees, "Molecular Mechanisms of Intrinsic Skin Aging and Retinoid-Induced Repair and Reversal," Journal of Investigative Dermatolology. Symposium Proceedings, Vol. 3, No. 1, 1998, pp. 57-60.

[13] B. Woerle, C. W. Hanke and G. Sattler, "Poly-L-LacticAcid: A Temporary Filler for Soft Tissue Augmentation," Journal of Drugs in Dermatology, Vol. 3, No. 4, 2004, pp. 385-389.

[14] B. L. Eppley and B. Dadvand, "Injectable Soft-Tisue Fillers: Clinical Overview," Plastic and Reconstructive Surgery, Vol. 118, No. 4, 2006, pp. 98e-106e. doi:10.1097/01.prs.0000232436.91409.30

[15] R. N. Sherman, "Sculptra: The New Three-Dimensional Filler," Clinics in Plastic Surgery, Vol. 33, No. 4, 2006, pp. 539-550. doi:10.1016/i.cps.2006.09.001

[16] S. M. Lam, B. Azizzadeh and M. Graivier, "Injectable Poly-L-Lactic Acid (Sculptra): Technical Considerations in Soft-Tissue Contourning," Plastic and Reconstructive Surgery, Vol. 118, No. 3, 2006, pp. 55S-63S. doi:10.1097/01.prs.0000234612.20611.5a

[17] S. Gogolewski, M. Jovanovic, S. M. Perren, J. G. Dillon and M. K. Hughes, "Tissue Response and in Vivo Degradation of Selected Polyhydroxyacids: Polylactides (PLA), Poly(3-hydrroxubutarate) (PHB), and Poly(3-hydroxybutarate-co-3-hydroxyvalearte) (PHB/VA)," Journal of Biomedical Material Research, Vol. 27, No. 9, 1993, pp. 1135-1148. doi:10.1002/jbm.820270904

[18] D. Vleggaar, "Facial Volumetric Correction with Injectable Poly-L-Lactic Acid," Dermatologic Surgery, Vol. 31, No. 11 Pt 2, 2005, pp. 1511-1518.

[19] P. Engelhard, G. Humble and D. Mest, "Safety of Sculptra: A Review of Clinical Data," Journal of Cosmetic and Laser Therapy, Vol. 7, No. 3/4, 2005, pp. 201-205. doi:10.1080/14764170500451404

[20] T. Lombardi, J. Samson, F. Plantier, C. Husson and R. Küffer, "Orofacial Granulomas after Injection of Cosmetic Fillers. Histopathological and Clinical Study of 11 Cases," Journal of Oral Pathology \& Medicine, Vol. 33, No. 2, 2004, pp. 115-120. doi:10.1111/j.1600-0714.2004.00194.x

[21] J. C. Dallon and H. P. Ehrlich, "A Review of FibroblastPopulated Collagen Lattices," Wound Repair and Regeneration, Vol. 16, No. 4, 2008, pp. 472-479. doi:10.1111/j.1524-475X.2008.00392.x

[22] S. Prahl, T. Kueper, T. Biernoth, Y. Wöhrmann, A. Münster, M. Fürstenau, et al., "Aging Skin Is Functionally Anaerobic: Importance of Coenzyme Q10 for Anti Aging Skin Care," BioFactors, Vol. 32, No. 1-4, 2008, pp. 245255. doi:10.1002/biof.5520320129

[23] H. Levinson, K. E. Koyer, G. C. Saggers and H. P. Ehrlich, "Calmodulin-Myosin Light Chain Kinase Inhibition Changes Fibroblast-Populated Collagen Lattice Contrac- 
tion, Cell Migration, Focal Adhesion Formation, and Wound Contraction," Wound Repair and Regeneration, Vol. 12, No. 5, 2004, pp. 505-511. doi:10.1111/j.1067-1927.2004.012502.x

[24] R. S. Adelstein, M. D. Pato, J. R. Sellers, P. de Lanerolle and M. A. Conti, "Regulation of Contractile Proteins by Reversible Phosphorylation of Myosin and Myosin Kinase," Society of General Physiologists Series, Vol. 37, 1982, pp. 273-281.

[25] A. B. Lansdown, "Calcium: A Potential Central Regulator in Wound Healing in the Skin," Wound Repair and Regeneration, Vol. 10, No. 5, 2002, pp. 271-285. doi:10.1046/j.1524-475X.2002.10502.x

[26] P. Carinci, M. Bodo, R. Evangelisti and E. Becchetti, "Involvement of the Cytoskeleton in Regulation of Collagen Synthesis and Secretion," Italian Journal of Anat- omy and Embryology, Vol. 100, Suppl. 1, 1995, pp. 6574.

[27] S. K. Donaldson, L. Hermansen and L. Bolles, "Differential, Direct Effects of $\mathrm{H}^{+}$on $\mathrm{Ca}^{2+}$-Activated Force of Skinned Fibres from the Soleus, Cardiac and Adductor Magnus Muscles of Rabbits," Pflugers Archive, Vol. 376, No. 1, 1978, pp. 55-65. doi:10.1007/BF00585248

[28] N. J. Kruse and P. Bornstein, "The Metabolic Requirements for Transcellular Movement and Secretion of Collagen," The Journal of Biological Chemistry, Vol. 250, No. 13, 1975, pp. 4841-4847.

[29] K. R. Beer and M. I. Rendon, "Use of Sculptra Mark in Esthetic Rejuvenation," Seminars in Cutaneous Medicine and Surgery, Vol. 25, No. 3, 2006, pp. 127-131. doi:10.1016/j.sder.2006.06.004 